\title{
Conical Coaxial Capacitors and Their Advantages
}

\author{
M. C. Selby \\ (June 16, 1959)
}

\begin{abstract}
Adjustable capacitors having electrodes in the form of coaxial cones or frustums have been used on rare occasions in the past, but their potential superiority to other types of capacitors for some important applications has been overlooked. The advantage of this geometry over cylindrical or disk forms is that the practical capacitance range is several times larger. An example cites the capacitance ranges for the same mechanical and percentage accuracy of a disk, cylindrical and conical type to be 10, 40, and 168 to 1 , respectively. An approximate equation was derived for this conical capacitor and close agreement is shown between computed and measured values of capacitance versus electrode displacement. Multiple cone and specially shaped electrodes are suggested to obtain large values of capacitance with an appreciable saving of space and further increased range of capacitance.
\end{abstract}

\section{Introduction}

Adjustable capacitors of coaxial form are frequently more suitable for a given application than the conventional rotary or compression types. ${ }^{1}$ Variation of capacitance in the former is obtained by linear displacement of one electrode, usually a cylinder or a disk, with respect to another similar stationary electrode. Unfortunately, cylindricaland disk-type electrodes have a relatively limited capacity range. Conical electrodes are intuitively attractive because they represent an intermediate case between cylindrical and disk electrodes. So far as the author is aware, no expression for the capacity of a conical structure has been derived. Such an expression is given below and will be of help in determining the effect of the dimensions and angle of the cone on capacity values and particularly on the range of capacity. ${ }^{2}$ This range is affected primarily by two things, the mechanical presetability and end effects. The first determines the minimum or maximum capacity which can be preset to a desired accuracy; this limiting factor seems to be the more important of the two and will be treated below.

- 'The end effect limits the minimum capacity and will be neglected here. Unfortunately, this effect is rather difficult to compute or to estimate. In neglecting it we favor the cylindrical case where this effect is more pronounced than in the other two cases. It will be shown that the conical capacitor has a considerably wider range even if the end effect - is neglected.

Let us assume a certain given cylindrical electrically shielded space within which an adjustable capacitor is to be placed. The shield is usually grounded. In cylindrical geometry the capacitance is directly proportional to the length of the meshed sections. If we are to be certain that all preset - capacities are accurate to, say, \pm 0.2 percent we \footnotetext{
$1 \mathrm{~J}$. C. Balsbaugh and P. H. Moon, A bridge for precision power-facto
measurements on small oil samples, Trans. AIEE 52, 528 (June 1933). measurements on small oil samples, Trans. AIEE 52, 528 (June 1933).
2 M. C. Selby, Analysis of coaxial conical capacitor (publication pending).
}

must make sure that the position of the traveling electrode is always reproduced at least to 0.2 percent; the critical position will, of course, be at the minimum capacity. For a micrometer having a maximum travel of $2 \mathrm{in}$. and a resetability of \pm 0.1 mil everywhere along this travel, the smallest meshed section will have to be 50 mils. The ratio of maximum to minimum capacity will therefore be 40 to 1 .

On the other hand, for practical purposes, a disktype capacitor ceases to behave like a normal capacitor approximately at separations exceeding the value of the radius of the disks. At higher separations these disks placed inside a shield have relatively high shunt capacities to the shield. Let us consider in all cases 1 in. as the diameter of the available cylindrical space; the shield may be $1 \frac{11}{2}$ or 2 in. in diameter. The critical position of the micrometer in the case of disks will be at the maximum capacity, namely at a disk separation of 50 mils. The range will therefore be fixed approximately by the ratio of one-half in. to 50 mils or 10 to 1 . With conical electrodes one can realize a range many times that of a cylindrical or disk capacitor.

\section{Conical Capacitor}

Consider a capacitor formed by two right frustums as shown in figure 1 . The cross section shows the essential dimensional elements of this capacitor. Let the sections of the conical electrode surfaces indicated by the length $l$ be referred to as the "meshed" surfaces. Let also:

$2 \theta=$ angle of the cones.

$D=$ adjustable distance between the apexes of the cones. As $D$ increases the capacitance decreases, because the distances between the conical surfaces increase and the length of meshed surfaces is reduced.

$h=$ the height of the inside cone $(A)$ from its apex to the base of its effective meshed surface. 


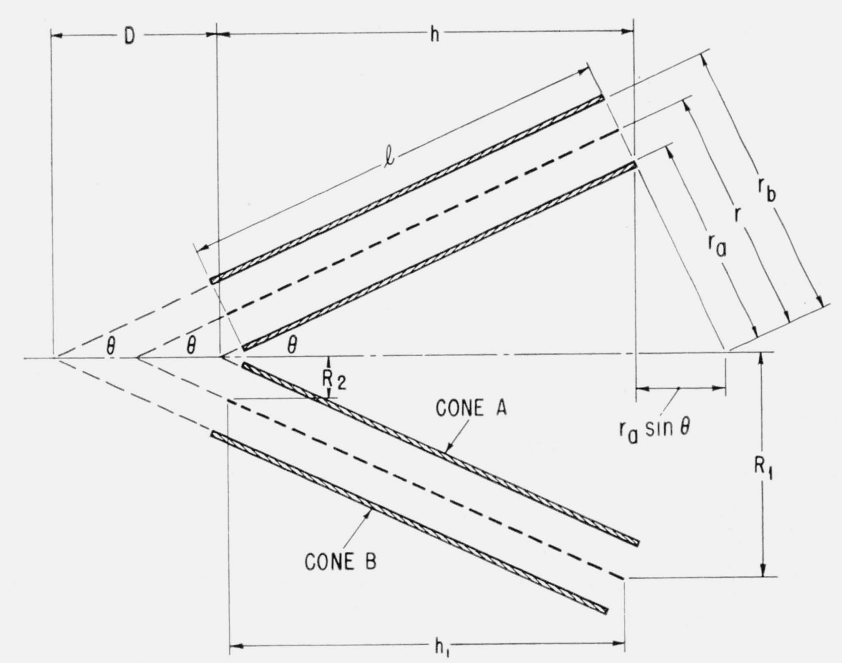

Figure 1. Cross-sectional dimensions of conical capacitor.

Solid lines indicate the conducting surfaces of frustums $A$ and $B$, respectively. Heavy dashed lines indicate a surface of a frustum taken at random parallel to the two conducting surfaces. The thin dashed lines indicate the geometric extention of the 3 surfaces to the apexes of the cones.

A rigorous analytical solution for the conical case seems, unfortunately, unobtainable at this time. The classical approach and existing limitations to the particular boundary value problem were investigated and the results (not shown here) were discouraging. In order to develop more confidence in an approximate solution, one of the field construction methods, the graphical, was employed to justify the basic introductory assumption that the charge distribution over the conical surfaces is essentially uniform. A physical visualization of two closelyspaced parallel conducting closed surfaces of any shape may be of further assistance in this respect. Assuming a certain potential of the inner conductor and zero potential on the outer, the charge distribution over the inner will be a normal function of the contour when the spacing between these conductors is infinity. As this spacing is reduced to zero the surface charges will be redistributed and will approach uniformity. At certain finite, and particularly at relatively small spacings, uniform charge distribution may be assumed with fairly good accuracy. The potential gradient is therefore perpendicular to the two metal surfaces nearly everywhere in the space between them. Since the conical conducting surfaces are parallel and coaxial, it follows that the electric lines in the dielectric space (assumed to be air) are everywhere normal to both surfaces. Moreover, since the electrodes are equipotential surfaces, it follows also that everywhere over these surfaces and over any other surface parallel to and located between these conducting surfaces

$$
\mathbf{E} \cdot \boldsymbol{\eta}=\mathbf{E}=-\frac{d V}{d n}
$$

where $\mathbf{E}$ is the potential gradient or the electric field intensity, $\boldsymbol{\eta}$ is unit vector normal to the surfaces, and $V$ is the potential at any point.
When relative positions and distances between the cones increase and begin to approach the order of magnitude of the height of the frustums, the wellknown condition is being approached when $\sigma$ is a maximum near the apex of the inside cone and a minimum near the inside apex of the enclosing cone.

Referring to figure 1 and taking the surface $S_{f}$ of any other coaxial frustum of half angle $\theta$ located between the two conical electrodes and applying Gauss' law to this surface, the total enclosed charge $Q$ is given by

$$
\int_{S} \mathbf{D} \cdot d \mathbf{S}=\epsilon E S_{f}=Q
$$

The dielectric constant of the interelectrode space is $\epsilon$, and the integral is applied only to the conical surface because the surfaces of the bases of the frustum by assumption do not contribute any electric flux.

From the geometry of the figure and the wellknown interrelations of $C=Q / V$

$$
\begin{aligned}
V & =-\int_{r_{b}}^{r_{a}} E d r=-\frac{Q}{\epsilon \pi l} \int_{r_{b}}^{r_{a}} \frac{d r}{(2 r \cos \theta-l \sin \theta)} \\
& =\frac{Q}{2 \epsilon \pi l \cos \theta} \ln \frac{\left(2 r_{b} \cos \theta-l \sin \theta\right)}{\left(2 r_{a} \cos \theta-l \sin \theta\right)} .
\end{aligned}
$$

Expressing $r_{a}$ and $r_{b}$ in terms of $h$ and $D$ we have

$$
C=\frac{2 \pi \epsilon l \cos \theta}{\ln \left[1+\frac{2 D \cos ^{2} \theta}{2 h-l \cos \theta}\right]} .
$$

The values of $r, r_{a}$, and $r_{b}$ are defined in figure 1 .

When the internal electrode is a cone, it may be considered a frustum having a negligible small top section and an altitude $h=l \cos \theta$. Then

$$
C=\frac{2 \pi \epsilon h}{\ln \left[1+2(D / h) \cos ^{2} \theta\right]} .
$$

Using the absolute rationalized practical system of units, $h$ is in meters, and $\epsilon \cong 8.854 \times 10^{-12}$ farads per meter (in vacuum and approximately in air) and

$$
C=55.6 h \frac{1}{\ln \left[1+2(D / h) \cos ^{2} \theta\right]} p f .
$$

\section{Computed and Measured Values}

Figure 2 compares experimental data with calculations based on eq (6). $\theta=10^{\circ} 34^{\prime},(D+h)=2.845^{\prime \prime}$, height of enclosing cone $=2.845^{\prime \prime}$, and the maximum displacement was 2 in.

In order to compare the range of the conical capacitor with others, one must do so within the same mechanical precision limits, e.g., one must find the minimum value of $D$ consistent with a reproducibility 


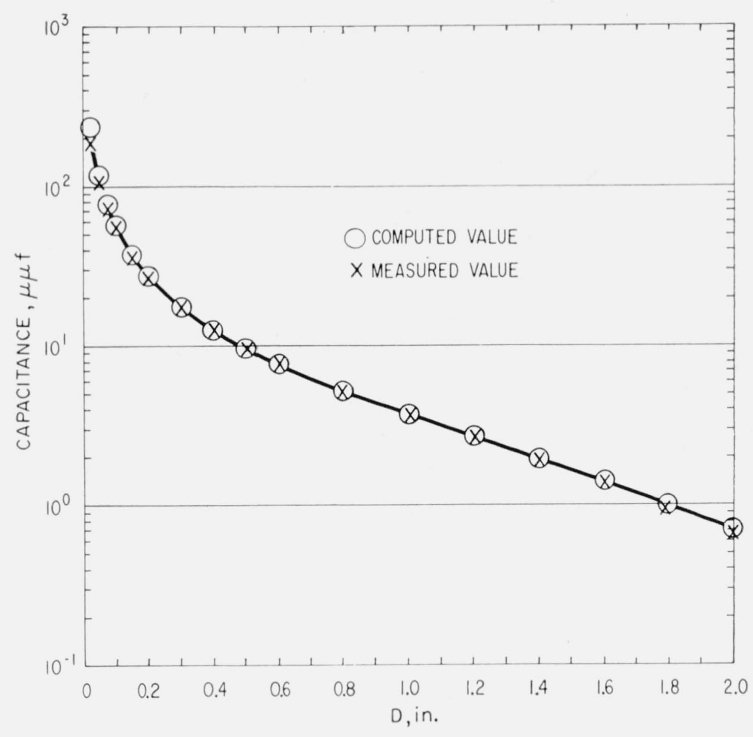

Figure 2. Capacitance values of right-cone continuously adjustable capacitor versus displacement.

of, say, 0.2 percent in capacitance, which is the precision considered above in the hypothetical cylindrical example. The derivative of $C$ with respect to $D$ or $h$ will yield the necessary information.

$$
\frac{d C}{C}=\frac{d h}{h}[1+F(h)]
$$

where

$$
F(h)=\frac{2(D+h) \cos ^{2} \theta}{\left(h+2 D \cos ^{2} \theta\right)\left[\ln \left(1+2 \frac{D}{h} \cos ^{2} \theta\right)\right]} .
$$

The error in the capacitance is thus larger than - that in $h$ by an additional factor $F(h)$, a function of $h$.

It is readily seen that $F(h)$ is a pure numeric and that $h$ may be used in any units. The table below gives one computed value of $F(h)$ and expected error for the capacitor of figure 2 for

$$
\frac{\Delta h}{h} \cong \frac{1}{3} \times 10^{-4} .
$$

TABLE 1.

\begin{tabular}{c|c|c|c|c|c|c}
\hline \hline$D^{\prime \prime}$ & $h^{\prime \prime}$ & $F(h)$ & $\Delta C / C$ & $C \max$. & $C \min$. & $\frac{C \text { max. }}{C \min .}$ \\
\hline 0.050 & 2.795 & 56 & $0.2 \%$ & 116.1 & 0.69 & 168 \\
\hline
\end{tabular}

It appears, therefore, that for the same resetability of $h$ as for the cylindrical and disk cases of 0.1 mil, a reproducibility of capacitance values to 0.2 percent, and a maximum displacement of $2 \mathrm{in}$., the range of the conical capacitor of the above dimensions would be about 168 to 1 , as against 40 to 1 for the cylindrical and 10 to 1 for the disk type. The conical type capacitor recently found practical application at the National Bureau of Standards in an attenuatorthermoelectric (AT) type $\mathrm{r}-\mathrm{f}$ voltmeter for voltages from an average of 5 to $1000 \mathrm{v}$ at frequencies of 1 to $10 \mathrm{Mc}^{3}$

Figure 2 shows satisfactory agreement between computed and measured capacitance values of an experimental conical capacitor. Validity of formula (6) was also established for $\theta=20^{\circ}$ and $45^{\circ}$ with good agreement between computed and measured values.

Several female and male conical conductors may be combined to form a single capacitor. Such a capacitor having three pairs of conductors is shown in figure 3. Capacitance ranges 2 to 300 or 3 to 450 ,

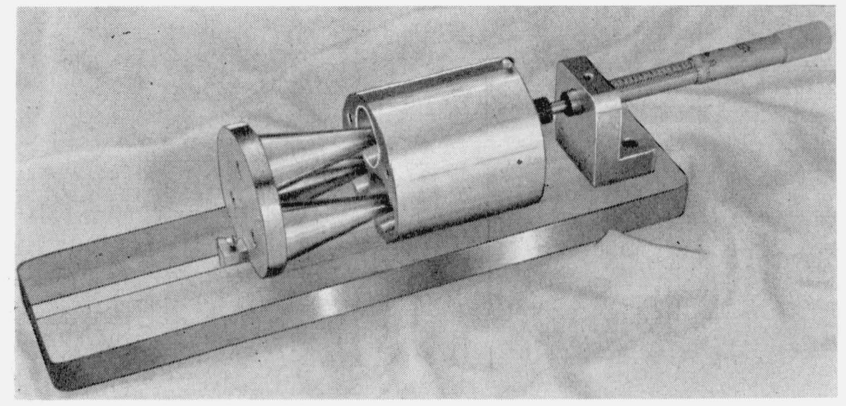

Figure 3. Conical coaxial capacitor employing three pairs of cones.

etc., pf may thus be obtained within practical space limitations. Moreover, longitudinal displacements of high accuracy at large values of $D$ are relatively easy to obtain; this enables the realization of still larger capacitance ranges by reducing say, the 3-pf minimum capacitance to $1 \mathrm{pf}$. It is evident that one can obtain different curves of capacitance versus displacement by changing the cross-sectional contour of one or both surfaces of the electrodes from conical to other suitable shapes. One should also be able to increase the range further by making use of additional ring-flanges at the bases of the cones; the cross-sectional contours of these rings may in turn be shaped to produce a certain desired function of capacitance versus displacement.

3 These types of voltmeters were announced and briefly described in NBS Tech. News Bull, 40-29 (1956).

Boulder, Colo.

(Paper 63C2-10) 